\title{
Hope or hype in the treatment of schizophrenia - what's the role of the physician?
}

\author{
Rodrigo A. Bressan, Geder E. M. Grohs, Gabriela Matos and Sukhi Shergill
}

\section{Summary}

According to the experience of people with schizophrenia, their psychiatrists' attitude towards the outcome of their illness is lacking in hope, which directly affects mutual faith in treatment. Here we discuss the scientific basis of hope and show its instrumental role in optimising the best treatment strategies for schizophrenia.

\section{Declaration of interest}

R.A.B has received honoraria for educational input and nonfinancial support from Ache; honoraria for educational input from Lundbeck; grants, honoraria for educational input and non- financial support from Janssen; all outside the submitted work. G.E.M.G. has received honoraria for educational input and non-financial support from Janssen outside the submitted work. G.M. reports support from Janssen-Cilag, outside the submitted work, and is an employee at Janssen-Cilag. S.S. has received grants and honoraria for educational input from EnVivo Pharmaceuticals, Takeda, AbbVie and Janssen Pharmaceuticals, outside the submitted work.

\section{Copyright and usage}

(C) The Royal College of Psychiatrists 2018
Rodrigo A. Bressan (pictured) is a visiting professor in the Department of Psychosis Studies at the Institute of Psychiatry, Psychology and Neuroscience at King's College London and also is a professor of psychiatry at PROESQ - Schizophrenia Program and LiNC (Interdisciplinary Laboratory of Clinical Neuroscience), Universidade Federal de São Paulo in Brazil. Geder E. M. Grohs is a a Preceptor Psychiatrist in the Schizophrenia Program at the Psychiatric Residency of IPqSC/SES - Santa Catarina, Brazil. Gabriela Matos is a scientific consultant at Janssen-Cilag Farmacêutica in São Paulo. Sukhi Shergill is Professor of Psychiatry \& Systems Neuroscience in the Department of Psychosis Studies at the Institute of Psychiatry, Psychology and Neuroscience in London.

\section{Introduction}

Psychiatrists' attitudes towards outcomes from schizophrenia are lacking in 'hope'; this is not only demoralising for patients and their families but limits the extent to which psychiatrists utilise the evidence-based interventions that hold promise for improving outcomes - using clozapine, long-acting injectable (LAI) antipsychotic medications and psychosocial interventions. This seems a controversial statement that most psychiatrists would disagree with, however, the experience of mental health users is that professionals often have a negative perspective of schizophrenia that they suggest directly affects them, making them less hopeful. ${ }^{1}$ This lack of hope is evidenced by the failure to offer these treatment options to patients, thus, denying patients the potential benefits of these treatments and, indeed, contributes to a self-fulfilling prophecy of poor outcomes. Here we examine the basis of this biased view of outcomes in schizophrenia, that directly influence psychiatrists' own 'hope' towards the prognosis and impact has on their decisions concerning pharmacological and psychological therapy. We also discuss the impact of 'hype' related to new discoveries in the field, which in the long term may have a deleterious effect on treatment.

\section{Hope theory}

What is hope? In the medical literature, the term 'hope' is frequently cited as a fundamental element for a successful treatment. ${ }^{1,2}$ Hope has many definitions including a positive perspective of the future; the expectation of achieving an objective; an effective coping strategy; an inner power that enables one to overcome obstacles (for a review see Snyder $e t a l^{3}$ ). As originally theorised by Snyder $e t ~ a l,{ }^{3}$ the 'hope theory' refers to an individual's positive perspective that make them invest energy and planning toward goal attainment. Hope is often confused with optimism; optimism or 'hype' is related to the individual's general expectancy for good rather than bad outcomes in their life. The hope theory intends to assess one's capacity to select appropriate routes and overcome barriers to goals rather than just one's confidence in a positive outcome. This can best be exemplified by the following analogy - an optimistic person would expect no rain and therefore leave home without an umbrella, whereas the hopeful person would expect no rain but take an umbrella to cope with any unexpected rain. How can one evidence the importance of hope? One could examine data from diseases where it is possible to have biological measures of outcomes - an example would be diabetes mellitus. Van Allen et al ${ }^{2}$ prospectively examined the associations between patients' hope and optimism with health outcomes in a sample of young people with type 1 diabetes mellitus; they found that hope improved glycaemic control whereas optimism did not help - and they are developing interventions to improve patients' and families' hope. In psychiatry, only the early intervention programmes for psychosis have incorporated hope as a treatment target and has been presented as an important example in the field.

\section{Psychiatrists' attitudes to schizophrenia}

Although hope is a key element for recovery, ${ }^{1}$ how many psychiatrists have a truly positive perspective of outcomes in schizophrenia? Schizophrenia has traditionally been viewed as a neurodegenerative chronic condition with a very pessimistic outlook. The tautological approach in which the diagnosis of schizophrenia is questioned when there is complete return to premorbid functioning still prevails. Perhaps it is not surprising that psychiatrists display higher scores of negative stereotypes of schizophrenia and perceived prejudice than the general population. ${ }^{4}$

So why is it that clinicians still have a negative perspective of schizophrenia? According to Cohen \& Cohen, ${ }^{5}$ psychiatrists suffer from 'the clinician's illusion' - since the patient population who seek specialised treatment are chronic, more severe and have 
more comorbidities, which per se presents the worst outcome of the disease, however, this group is not representative of the range of schizophrenia. Clearly, sustaining a positive perspective about treatment effectiveness in this scenario is very challenging; given this negative bias it is difficult for clinicians to remain hopeful and focus energy and planning towards the best treatment strategies available for each patient.

\section{Translation of current knowledge into clinical care}

What opportunities for change have arisen recently? There have been significant advances in our understanding of schizophrenia; there is a wealth of clinical and neurobiological data demonstrating that schizophrenia is a neurodevelopmental disorder with heterogeneous phenotypes and a markedly diverse range of outcomes. ${ }^{4}$ Similarly, recent large cohort studies have shown that the evolution of the disease is more favourable than previously thought. ${ }^{4}$

What are the best treatment strategies for people with schizophrenia? Although, the hype of neurobiological research has not yet provided a reliable set of predictors of the disease trajectory, there is robust evidence showing that antipsychotic medications and psychosocial interventions are fundamental elements in improving outcomes in schizophrenia. ${ }^{6}$ In order to discuss the implication for clinical attitudes, we focus on three aspects of the treatment of schizophrenia that are recommended in the majority of the guidelines, including those of the American Psychiatric Association and the UK National Institute for Health and Care Excellence (NICE), namely: (a) patients whose recovery is limited by poor adherence should be offered a trial of LAI; (b) patients who respond poorly to first- and second-line antipsychotics (treatment resistant) should be encouraged to have a trial of clozapine, and (c) patients with persistent symptoms and/or poor social interactions should be offered adjunct psychosocial treatments

Surprisingly, despite the clear advantages offered by LAIs, clozapine and psychosocial interventions, their use remains limited. ${ }^{7-10}$ Although non-adherence reaches about $50 \%$ in the first year of treatment in first-episode psychosis and partial adherence rates range from one- to two-thirds, LAIs are widely underused in many settings around the world. ${ }^{7}$ Rates of treatment resistance are also very high $(\sim 30 \%)$ and surveys show low rates of clozapine prescription ranging from 2 to $3 \%$ in North America to $15.9 \%$ in China and some European countries. ${ }^{8}$ Likewise, uptake of psychosocial treatments remains very low even though at least eight different evidence-based psychosocial interventions such as cognitive-behavioural therapy, family-based services and skills training are recommended by experts and people with the disorder. ${ }^{9}$

Why are clinicians not able to implement these evidence-based strategies that offer 'realistic hope' to people with schizophrenia? Modern care delivery processes involve an alliance between clinicians, the person with schizophrenia and their family and carers; this is a move away from paternalistic relationships towards more effective strategies, involving shared decision-making. ${ }^{1}$ Doctors have a key role in providing information and support in discussing LAIs, clozapine and psychosocial interventions to empower patients and caregivers in their decision-making. However, there can be logistical issues with the (un)availability of both pharmacological and psychosocial interventions, which it is argued, may be more to do with service resources than with hope, nevertheless, addressing resourcing issues also requires a positive attitude and hope for a positive outcome.

The current negative perspective towards the prognosis of schizophrenia is one example of poor translation of current knowledge to clinical care. Without a positive attitude it is difficult for clinicians to maintain 'hope'; creating a self-fulfilling prophecy, in which a false assessment of the situation evokes a behaviour that makes the original false conception come true. In other words, a biased view of the likely outcome in schizophrenia makes the doctors less hopeful towards the patients' prognosis and thus has an impact on their prescribing - precluding patients from access to the best treatment options. Inevitably, the lack of the most appropriate treatment has an impact on patients, resulting in poorer functioning and hindering the recovery process. These poor outcomes validate the original hopeless bias, perpetuating the reign of error! In practice, psychiatrists end up citing this course as proof that they were right to be hopeless about the poor prognosis of schizophrenia from the very beginning.

\section{Conclusions}

In summary, hope is a construct that incorporates the positive perspective, and more importantly, the energy, planning and actions to overcome the issues related to schizophrenia. Since 'hope' is not a static trait, it will have a different frame in each stage of the disease. To maintain a hopeful approach in more severe cases and chronic situations, clinicians need to better understand that people with schizophrenia are more interested in outcomes such as dependency, poor control of their own life and hopelessness than in symptom reduction. ${ }^{1}$ A hopeful attitude towards schizophrenia is not a naive optimistic approach, as in current educational thinking a growth mind-set ${ }^{10}$ advocating 'realistic hope' is essential for clinicians to provide state-of-the-art clinical care for people with schizophrenia.

Rodrigo A. Bressan, MD, PhD, Department of Psychosis Studies, Institute of Psychiatry, Psychology and Neuroscience, King's College London, UK, PROESQ - Schizophrenia Program, Universidade Federal de São Paulo, São Paulo and LiNC - Interdisciplinary Laboratory of Clinical Neurosciences, Universidade Federal de São Paulo, São Paulo, Brazil; Geder E. M. Grohs, MD, PhD, Schizophrenia Program, Psychiatric Residency of IPgSC/SES - Santa Catarina, Brazil; Gabriela Matos, PhD, Janssen-Cilag Farmacêutica, São Paulo, Brazil; Sukhi Shergill, MBBS, SFHEA, PhD, FRCPsych, Department of Psychosis Studies, Institute of Psychiatry, Psychology and Neuroscience, King's College London, UK

Correspondence: Rodrigo A. Bressan, Department of Psychosis Studies, Institute of Psychiatry, Psychology and Neuroscience King's College London, De Crespigny Park, London SE5 8AF UK. Email: rodrigo.bressan@kcl.ac.uk

First Received 2 May 2017, final revision 09 June 2017, accepted 27 June 2017

\section{Funding} R.A.B is supported by Fundacao de Amparo a Pesquisa do Estado de São Paulo (FAPESP 2016/
02246-5), Brasil, Conselho Nacional de Desenvolvimento Científico e Tecnológico (CNPq), Brasil, and Coordenação de Aperfeiçoamento de Pessoal de Nível Superior (CAPES), Brasil. S.S. is supported by a European Research Council Consolidator Award (Grant Number 311686) and the National Institute for Health Research (NIHR) Mental Health Biomedical Research Centre at South London and Maudsley NHS Foundation Trust and King's College London. None of the funders had any role in study design, data collection, data analysis, data interpretation, or writing of the report.

\section{References}

1 Bellack AS. Scientific and consumer models of recovery in schizophrenia: concordance, contrasts, and implications. Schizophr Bull 2006; 32: 432-42.

2 Van Allen J, Steele RG, Nelson MB, Peugh J, Egan A, Clements M, et al. A Longitudinal examination of hope and optimism and their role in type 1 diabetes in youths. J Pediatr Psychol 2016; 41: 741-9.

3 Snyder CR, Irving IM, Anderson JR. Hope and health. In Handbook of Social and Clinical Psychology: The Health Perspective (eds CR Snyder and DR Forsyth): 285-305. Pergamon Press, 1991.

4 Schulze B. Stigma and mental health professionals: a review of the evidence on an intricate relationship. Int Rev Psychiatry 2007; 19: 137-55.

5 Cohen P, Cohen J. The clinician's illusion. Arch Gen Psychiatr 1984; 41: 1178-82.

6 Tandon R, Nasrallah HA, Keshavan MS. Schizophrenia, 'just the facts' 5. Treatment and prevention. Past, present, and future. Schizophr Res 2010; 122: $1-23$. 
7 Patel MX, Haddad PM, Chaudhry IB, McLoughlin S, Husain N, David AS. Psychiatrists' use, knowledge and attitudes to first- and second-generation antipsychotic longacting injections: comparisons over 5 years. J Psychopharmacol 2010; 24: 1473-82.

8 Gören JL, Meterko M, Williams S, Young GJ, Baker E, Chou CH, et al. Antipsychotic prescribing pathways, polypharmacy, and clozapine use in treatment of schizophrenia. Psychiatr Serv 2013; 64: 527-33.
9 Dixon LB, Dickerson F, Bellack AS, Bennett M, Dickinson D, Goldberg RW, et al. Schizophrenia Patient Outcomes Research Team (PORT). The 2009 schizophrenia PORT psychosocial treatment recommendations and summary statements. Schizophr Bull 2010; 36: 48-70.

10 Dweck CS, Leggett EL. A social-cognitive approach to motivation and personality. Psychol Rev 1998; 95: 256-73.

\section{Psychiatric Disorder among the Yoruba by Alexander H. Leighton, T. Adeoye Lambo, Charles C. Hughes, Dorothea C. Leighton, Jane M. Murphy and David B. Macklin (Cornell University Press, 1963)}

\section{Niran Okewole}

There are a number of reasons why one might revisit a book published 54 years ago. At the time of publication, Psychiatric Disorder among the Yoruba provided a strong rebuttal of many of the prevailing ideas regarding the 'African mind'. Ethnopsychiatry, the predominant theoretical framework of the colonial era, which governed the way the mind of 'the African' was perceived by the settler colonials, had held sway for the previous half century. Against the poorly grounded theorising of supposed experts such as Carothers, as well as others at different points along the spectrum of expertise, the Cornell-Aro project sought to provide empirical data regarding the mental health of an African community.

The methodological approach, which was a combination of psychiatric and sociological/anthropological investigation, was also novel at the time. Thitherto, there had been little or no cross-talk between psychiatrists and sociologists, which nevertheless did not prevent them from making sweeping generalisations. African communities were largely viewed as 'primitive' cultures and theorised as such.

One key strength of this work is the rigour with which the question of cultural distortion is approached. It bears remembering that this was mid-20th century, and the descriptive, phenomenological approach of Jaspers was engaged in mortal combat with the theory-driven postulates of psychoanalysis. In any case, anthropologists like Malinowski claimed that having investigated some 'primitive' cultures, they found no evidence for the Oedipus complex there. If a universal, atheoretical language of psychiatry was to gain ascendancy, signs and symptoms had to be the same across cultures. The collaborative effort - the objective could not have been achieved otherwise - between psychiatrists and sociologists, Yoruba and American, generated previously unavailable data which helped fuel a paradigm shift: the investigators found that the identification of symptoms of mental illness (with the exception of entities such as phobias and obsessive-compulsive disorder) among the Yoruba was considerably similar to European and American populations. Although there were some similar ideas about causation (drugs and medicines, heredity, contagion, physical and psychological trauma), the Yoruba gave prominence to other ideas (notably malignant influences, superhuman and human, violation of one's destiny, and fate). These ideas persist to this day, and have only been amplified by Christianity and Islam.

Besides the obvious debt to Emil Durkheim's concept of anomie, a current of thought prominent at the time of publication, against which the book can also be read, is Fanonism. While the work of Leighton et al was contemporaneous with the publications of Fanon, one benefit of reading it half a century later is that it is possible to see the lines of intersection. Leighton et al sought to investigate the concept of social integration/disintegration as an environmental risk factor for mental illness. Having found the same in the Stirling County study, the authors reported a higher prevalence of mental disorders in disintegrated Yoruba communities. Indeed, they suggested that the less integrated communities in the American and Yoruba studies had more in common with each other than their better integrated neighbouring communities. This fortuitously can be read into the Fanonist framework, if one views the impact of colonialism as disruptive of the colonised communities. Several studies since then have reported disparate prevalence, especially along the rural/urban dichotomy. A contemporary area of deficit however is the ploughing back of these data into psychosocial theory.

On the shelves today there is hardly any work to match the scope or address the questions raised over half a century ago by Leighton et al. Nor is there likely to be. Leighton et al, while seeking to entrench a methodology of objective phenomenological enquiry, were beneficiaries of a culture of close observation. This close clinical scrutiny is gradually being de-emphasised in the training of psychiatrists. At the same time, as biological psychiatry gains ascendancy, the chasm between psychiatry and sociology/anthropology may well be widening. Knowledge production as an interdisciplinary enterprise is left the poorer.

The authors acknowledged their limitations, some of which were limitations of the state of psychiatry itself. Nevertheless, they managed to produce a landmark volume which stands as a gentle rebuke in our time. 